\title{
Crystal chemical models of point defects in compounds of the Pb-Cr-Te system
}

\author{
Dmytro FREIK $^{1}{ }^{*}$, Liliya TUROVSKA ${ }^{1}$, Lyubov MEZHYLOVSKA ${ }^{1}$, Volodymyra BOYCHUK ${ }^{1}$ \\ ${ }^{1}$ Institute of Physics and Chemistry, Precarpathian National Vasyl Stefanyk University, \\ Shevchenka St. 57, 76018 Ivano-Frankivsk, Ukraine \\ * Corresponding author. Tel.: +380-34-2759214; fax:+380-34-2759214; e-mail: freik@ pu.if.ua
}

Received April 14, 2010; accepted June 29, 2010; available on-line November 5, 2010

Models describing point defects in chromium doped lead telluride crystals and in the solid solutions PbTeCrTe and $\mathrm{PbTe}_{-} \mathrm{Cr}_{3} \mathrm{Te}_{4}$ have been developed. Based on the proposed quasi-chemical formulae, the dependence of the concentration of defects and free carriers, and the Hall concentration of current carriers, on the content of dopant and the deviation from PbTe stoichiometry, have been calculated. The mechanisms of doping and solid solution formation have been analyzed.

Lead telluride / Solid solutions / Point defects / Quasi-chemical formulae

\section{Кристалохімічні моделі точкових дефектів у сполуках системи $\mathrm{Pb}-\mathrm{Cr}-\mathrm{Te}$}

\author{
Дмитро ФРЕЇК ${ }^{1 *}$, Лілія ТУРОВСЬКА ${ }^{1}$, Любов МЕЖИЛОВСЬКА ${ }^{1}$, Володимира БОЙЧУК ${ }^{1}$ \\ ${ }^{1}$ Фізико-хімічний інститут, Прикарпатський національний університет імені Василя Стефаника, \\ вул. Шевченка 57, 76018 Івано-Франківськ, Украӥна \\ * Контактна особа. Тел.: +380-34-2759214; факс:+380-34-2759214; e-mail: freik@pu.if.ua
}

Розроблені моделі точкових дефектів у легованих хромом кристалах плюмбум телуриду, а також твердих розчинів PbTe-CrTe та $\mathrm{PbTe}_{\mathrm{C}} \mathrm{Cr}_{3} \mathrm{Te}_{4}$. На основі запропонованих кристалоквазіхімічних формул розраховано залежності концентрації дефектів, вільних носіїв, холлівської концентрації носіїв заряду від вмісту легуючих сполук і відхилення від стехіометрії у РbТе. Визначено механізми легування та утворення твердих розчинів.

Плюмбум телурид / Тверді розчини / Точкові дефекти / Кристалоквазіхімічні формули

Вступ

Напівпровідникові сполуки IV-VI є базовими матеріалами для створення термоелектричних перетворювачів енергії в середній області температур (500-750) К, а також приймальних i випромінювальних структур інфрачервоного діапазону оптичного спектру [1]. Плюмбум телурид завдяки своїм фізико-хімічним властивостям займає особливе місце серед цих сполук [1,2]. Так, зокрема, він володіє вузькою двосторонньою областю гомогенності, яка визначає n- або p-тип провідності i складний спектр власних точкових дефектів [3,4].

На даний час, незважаючи на значну кількість експериментальних досліджень та теоретичних розрахунків [1-3], питання, пов'язані із кристалохімією точкових дефектів, їх зарядовим станом як у чистих, так і легованих кристалах $\mathrm{PbTe}$, а також твердих розчинах на його основі, залишаються дискусійними. Це значною мірою гальмує отримання матеріалу із наперед заданими властивостями та перспективу його практичного використання. 
У пропонованій роботі досліджено природу точкових дефектів у кристалах потрійної системи $\mathrm{Pb}-\mathrm{Cr}-\mathrm{Te}$ на основі плюмбум телуриду та їх вплив на комплекс фізико-хімічних властивостей.

\section{Фізико-хімічні властивості}

Легування $\mathrm{PbTe}$ хромом дозволяє отримати матеріал із надзвичайними параметрами, які пов’ язані зі збереженням магнітних властивостей до кімнатних температур і не вимагають глибокого охолодження, як халькогенідні шпінелі [5]. Так, зокрема, введення хрому зумовлює зменшення пікнометричної густини і параметра гратки [6], що підтверджує заміщення плюмбуму в катіонній підгратці і переважання вакансій. Дефекти заміщення $\mathrm{Cr}_{\mathrm{Pb}} €$ донорами, оскільки введення хрому збільшує концентрацію електронів у порівнянні 3 нелегованими кристалами. Це підтверджується результатами двотемпературного відпалу кристалів PbTe:Cr у парі телуру $P_{\mathrm{Te}_{2}}$ [6]: величина парціального тиску телуру, що відповідає термодинамічному n-p-переходу, зміщена на боці більших значень $P_{T e_{2}}$ відносно до чистого РbTе.

Область розчинності хром телуриду в $\mathrm{PbTe}$ незначна і складає лише 1 мол. \% при $500 \mathrm{~K}$ i 2 мол. \% при $1100 \mathrm{~K} \quad[7,8]$. При цьому із збільшенням вмісту CrTe параметр гратки i концентрація носіїв зменшуються, а питомий опір і коефіцієнт термо-е.p.c. зростають [8].

Сплави системи $\mathrm{PbTe}-\mathrm{Cr}_{3} \mathrm{Te}_{4}$ вивчали методами диференційно-термічного, мікроструктурного i рентгенофазового аналізів i вимірювали електрофізичні параметри зразків в інтервалі 0-70 мол. \% $\mathrm{Cr}_{3} \mathrm{Te}_{4}$ [9]. При $1100 \mathrm{~K}$ розчинність $\mathrm{Cr}_{3} \mathrm{Te}_{4}$ в $\mathrm{PbTe}$ мала і складає 0,5 мол. \%. Після досягнення межі розчинності сплави складаються 3 суміші $\mathrm{Cr}_{3} \mathrm{Te}_{4}$ та твердого розчину $\mathrm{PbTe}-\mathrm{Cr}_{3} \mathrm{Te}_{4}$ (система квазібінарна). При збільшенні вмісту $\mathrm{Cr}_{3} \mathrm{Te}_{4}$ (при малих його кількостях) параметр гратки зменшується. Після досягнення межі розчинності він практично не змінюється. Встановлено, що зі збільшенням вмісту $\mathrm{Cr}_{3} \mathrm{Te}_{4}$ концентрація носіїв струму зменшується, питомий опір і термо-е.p.c. збільшуються; при досягненні межі розчинності спостерігається зворотній хід вказаних величин. Зразки володіють електронною провідністю.

Зауважимо, що як у наведених літературних джерелах [5-8], так і в ряді інших [10-12] не встановлені не тільки домінуючі точкові дефекти, а тим паче їх кількісні співвідношення.

\section{Кристалохімічні моделі і формули}

Нестехіометричний плюмбум телурид. В основу методу кристалоквазіхімічного аналізу на основі антиструктури покладено суперпозицію кристалоквазіхімічних кластерів базової і легуючої сполук [13]. Антиструктурою плюмбум телуриду є галеніт $\mathrm{V}_{\mathrm{Pb}}^{/ /} \mathrm{V}_{\mathrm{Te}}^{\bullet}$, де $\mathrm{V}_{\mathrm{Pb}}^{/ /}$i $\mathrm{V}_{\mathrm{Te}}^{\bullet \bullet}$ - двократно заряджені негативна вакансія плюмбуму i позитивна вакансія телуру, “”” i “•” - негативний i позитивний заряди відповідно.

Кристалоквазіхімічна формула n-PbTe за умови розупорядкування за Френкелем у катіонній підгратці має вигляд:

$$
\begin{aligned}
& (1-\alpha)\left\{\mathrm{Pb}_{\mathrm{Pb}}^{\mathrm{x}} \mathrm{Te}_{\mathrm{Te}}^{\mathrm{x}}\right\}+\alpha \mathrm{Pb}^{0} \rightarrow \\
& {\left[\mathrm{Pb}_{1-\alpha}^{\mathrm{x}}\right]_{\mathrm{Pb}}\left[\mathrm{Te}_{1-\alpha}^{\mathrm{x}}\right]_{\mathrm{Te}}\left(\mathrm{Pb}_{\alpha}^{\bullet}\right)_{\mathrm{i}}+\alpha \mathrm{e}^{\prime}}
\end{aligned}
$$

де $\alpha$ - величина початкового відхилення від стехіометрії на боці $\mathrm{Pb}$.

Кристалохімічна формула для p-PbTe:

$\mathrm{V}_{\mathrm{Pb}}^{\prime /} \mathrm{V}_{\mathrm{Te}}^{\bullet \bullet}+\mathrm{Te}^{0} \rightarrow \mathrm{V}_{\mathrm{Pb}}^{\prime} \mathrm{Te}_{\mathrm{Te}}^{\times}+\mathrm{h}^{\bullet} ;$

$(1-\beta)\left\{\mathrm{Pb}_{\mathrm{Pb}}^{\mathrm{X}} \mathrm{Te}_{\mathrm{Te}}^{\mathrm{X}}\right\}+\beta\left\{\mathrm{V}_{\mathrm{Pb}}^{\prime} \mathrm{Te}_{\mathrm{Te}}^{\times}+\right.$

$\left.\mathrm{h}^{\bullet}\right\} \rightarrow\left[\mathrm{Pb}_{1-\beta}^{\mathrm{X}} \mathrm{V}_{\beta}^{\prime}\right]_{\mathrm{Pb}} \mathrm{Te}_{\mathrm{Te}}^{\mathrm{X}}+\beta \mathrm{h}^{\bullet}$

де $\beta$ - величина початкового відхилення від стехіометрії на боці Те.

Леговані кристали PbTe:Cr. За умови, що хром може перебувати як у двозарядному, так i трьохзарядному станах, умова диспропорціювання матиме вигляд:

$\mathrm{Cr}^{2+} \rightarrow \mathrm{Cr}_{\mathrm{z}}^{2+}+\mathrm{Cr}_{1-\mathrm{z}}^{3+}+(1-\mathrm{z}) \mathrm{e}^{-}$

де $\mathrm{z}$ - коефіцієнт диспропорціювання зарядового стану домішки $(\mathrm{z} \approx 0,25)$.

При цьому, якщо $\mathrm{Cr}^{2+}$ буде нейтральним дефектом ( $\mathrm{Cr}^{2+} \rightarrow \mathrm{Cr}_{\mathrm{Pb}}^{0}$ ) у відношенні до катіонної підгратки $\mathrm{PbTe}$, то $\mathrm{Cr}^{3+}$ - зарядженим донором $\left(\mathrm{Cr}^{3+} \rightarrow \mathrm{Cr}_{\mathrm{Pb}}^{+}\right)$.

Легуючий кластер, враховуючи (3), матиме вигляд:

$\mathrm{V}_{\mathrm{Pb}}^{/ /} \mathrm{V}_{\mathrm{Te}}^{\bullet \bullet}+\mathrm{Cr} \rightarrow \mathrm{V}_{\mathrm{Pb}}^{\prime /} \mathrm{V}_{\mathrm{Te}}^{\bullet \bullet}+\mathrm{Cr}_{\mathrm{z}}^{\bullet \bullet}+$

$\mathrm{Cr}_{1-\mathrm{z}}^{\bullet \bullet \bullet}+(3-\mathrm{z}) \mathrm{e}^{\prime} \rightarrow\left[\mathrm{Cr}_{1-\mathrm{z}}^{\bullet} \mathrm{Cr}_{\mathrm{z}}^{\times}\right]_{\mathrm{Pb}} \mathrm{V}_{\mathrm{Te}}^{\bullet \bullet}$

$(3-\mathrm{z}) \mathrm{e}^{\prime}$

Отже, кристалоквазіхімічна формула n-PbTe:Cr буде записана таким чином:

$$
\begin{aligned}
& (1-\mathrm{x})\left\{\left[\mathrm{Pb}_{1-\alpha}^{\mathrm{x}}\right]_{\mathrm{Pb}}\left[\mathrm{Te}_{1-\alpha}^{\mathrm{x}}\right]_{\mathrm{Te}}\left(\mathrm{Pb}_{\alpha}^{\bullet}\right)_{\mathrm{i}}+\right. \\
& \left.\alpha \mathrm{e}^{\prime}\right\}+\mathrm{x}\left\{\left[\mathrm{Cr}_{1-\mathrm{z}}^{\bullet} \mathrm{Cr}_{\mathrm{z}}^{\times}\right]_{\mathrm{Pb}} \mathrm{V}_{\mathrm{Te}}^{\bullet \bullet \bullet}+(3-\mathrm{z}) \mathrm{e}^{\prime}\right\} \rightarrow \\
& {\left[\mathrm{Pb}_{(1-\alpha)(1-\mathrm{x})}^{\mathrm{x}} \mathrm{Cr}_{(1-\mathrm{z}) \mathrm{x}}^{\bullet} \mathrm{Cr}_{\mathrm{Zx}}^{\times}\right]_{\mathrm{Pb}}\left[\mathrm{Te}_{(1-\alpha)(1-\mathrm{x})}^{\mathrm{x}} \mathrm{V}_{\mathrm{x}}^{\bullet \bullet}\right]_{\mathrm{Te}} \times} \\
& \left(\mathrm{Pb}_{\alpha(1-\mathrm{x})}^{\bullet}\right)_{\mathrm{i}}+\{\alpha+(3-\alpha-\mathrm{z}) \mathrm{x}\} \mathrm{e}^{\prime}
\end{aligned}
$$

де $\mathrm{x}$ - атомна частка легуючої домішки $\mathrm{Cr}$. 
Аналогічно для p-PbTe:Cr:

$(1-\mathrm{x})\left\{\left[\mathrm{Pb}_{1-\beta}^{\mathrm{x}} \mathrm{V}_{\beta}^{\prime}\right]_{\mathrm{Pb}} \mathrm{Te}_{\mathrm{Te}}^{\mathrm{x}}+\beta \mathrm{h}^{\bullet}\right\}+$

$\mathrm{x}\left\{\left[\mathrm{Cr}_{1-\mathrm{z}}^{\bullet} \mathrm{Cr}_{\mathrm{z}}^{\times}\right]_{\mathrm{Pb}} \mathrm{V}_{\mathrm{Te}}^{\bullet \bullet}+(3-\mathrm{z}) \mathrm{e}^{\prime}\right\} \rightarrow$

$\left[\mathrm{Pb}_{(1-\beta)(1-\mathrm{x})}^{\mathrm{x}} \mathrm{Cr}_{(1-\mathrm{z}) \mathrm{x}}^{\bullet} \mathrm{Cr}_{\mathrm{zx}}^{\times} \mathrm{V}_{\beta(1-\mathrm{x})}^{\prime}\right]_{\mathrm{Pb}}\left[\mathrm{Te}_{1-\mathrm{x}}^{\mathrm{x}} \mathrm{V}_{\mathrm{x}}^{\bullet \bullet}\right]_{\mathrm{Te}}+$

$\beta(1-\mathrm{x}) \mathrm{h}^{\bullet}+(3-\mathrm{z}) \mathrm{xe}^{\prime}$

Tвердi розчини PbTe-CrTe. Легуючий кластер, враховуючи (3), матиме вигляд:

$\mathrm{V}_{\mathrm{Pb}}^{\prime /} \mathrm{V}_{\mathrm{Te}}^{\bullet \bullet}+\mathrm{CrTe} \rightarrow\left[\mathrm{Cr}_{1-\mathrm{z}}^{\bullet} \mathrm{Cr}_{\mathrm{z}}^{\times}\right]_{\mathrm{Pb}} \mathrm{Te}_{\mathrm{Te}}^{\mathrm{x}}+$

$(1-\mathrm{z}) \mathrm{e}^{\prime}$

Отже, кристалоквазіхімічна формула $\mathrm{n}-\mathrm{Pb}_{1-\mathrm{x}} \mathrm{Cr}_{\mathrm{x}}$ Те буде записана таким чином:

$(1-\mathrm{x})\left\{\left[\mathrm{Pb}_{1-\alpha}^{\mathrm{x}}\right]_{\mathrm{Pb}}\left[\mathrm{Te}_{1-\alpha}^{\mathrm{x}}\right]_{\mathrm{Te}}\left(\mathrm{Pb}_{\alpha}^{\bullet}\right)_{\mathrm{i}}+\right.$

$\left.\alpha \mathrm{e}^{\prime}\right\}+\mathrm{x}\left\{\left[\mathrm{Cr}_{1-\mathrm{z}}^{\bullet} \mathrm{Cr}_{\mathrm{z}}^{\times}\right]_{\mathrm{Pb}} \mathrm{Te}_{\mathrm{Te}}^{\mathrm{x}}+(1-\mathrm{z}) \mathrm{e}^{\prime}\right\} \rightarrow$

$\left[\mathrm{Pb}_{(1-\alpha)(1-\mathrm{x})}^{\mathrm{x}} \mathrm{Cr}_{(1-\mathrm{z}) \mathrm{x}}^{\bullet} \mathrm{Cr}_{\mathrm{zx}}^{\times}\right]_{\mathrm{Pb}}\left[\mathrm{Te}_{(1-\alpha)(1-\mathrm{x})+\mathrm{x}}^{\mathrm{x}}\right]_{\mathrm{Te}} \times$

$\left(\mathrm{Pb}_{\alpha(1-\mathrm{x})}^{\bullet}\right)_{\mathrm{i}}+\{\alpha(1-\mathrm{x})+(1-\mathrm{z}) \mathrm{x}\} \mathrm{e}^{\prime} \rightarrow$

$\left[\mathrm{Pb}_{(1-\alpha)(1-\mathrm{x})}^{\mathrm{x}} \mathrm{Cr}_{(1-\mathrm{z}) \mathrm{x}}^{\bullet} \mathrm{Cr}_{\mathrm{zx}}^{\times}\right]_{\mathrm{Pb}}\left[\mathrm{Te}_{1-\alpha+\alpha \mathrm{x}}^{\mathrm{x}}\right]_{\mathrm{Te}}\left(\mathrm{Pb}_{\alpha(1-\mathrm{x})}^{\bullet}\right)_{\mathrm{i}}+$

$\{\alpha+(1-\alpha-z) x\} e^{\prime}$

де $\mathrm{x}$ - мольна частка CrTe.

Аналогічно для $\mathrm{p}-\mathrm{Pb}_{1-\mathrm{x}} \mathrm{Cr}_{\mathrm{x}} \mathrm{Te}$ :

$(1-\mathrm{x})\left\{\left[\mathrm{Pb}_{1-\beta}^{\mathrm{x}} \mathrm{V}_{\beta}^{\prime}\right]_{\mathrm{Pb}} \mathrm{Te}_{\mathrm{Te}}^{\mathrm{x}}+\beta \mathrm{h}^{\bullet}\right\}_{+}$

$\mathrm{x}\left\{\left[\mathrm{Cr}_{1-\mathrm{z}}^{\bullet} \mathrm{Cr}_{\mathrm{z}}^{\times}\right]_{\mathrm{Pb}} \mathrm{Te}_{\mathrm{Te}}^{\mathrm{x}}+(1-\mathrm{z}) \mathrm{e}^{\prime}\right\} \rightarrow$

$\left[\mathrm{Pb}_{(1-\beta)(1-\mathrm{x})}^{\mathrm{x}} \mathrm{Cr}_{(1-\mathrm{z}) \mathrm{x}}^{\bullet} \mathrm{Cr}_{\mathrm{zx}}^{\times} \mathrm{V}_{\beta(1-\mathrm{x})}^{\prime}\right]_{\mathrm{Pb}} \mathrm{Te}_{\mathrm{Te}}^{\mathrm{x}}+$

$\beta(1-\mathrm{x}) \mathrm{h}^{\bullet}+(1-\mathrm{z}) \mathrm{xe}^{\prime}$

тверді розчини $\mathrm{PbTe}_{-} \mathrm{Cr}_{3} \mathrm{Te}_{4}$. Відомо, що $\mathrm{Cr}_{3} \mathrm{Te}_{4}$ - брутто-формула, тому іï можна переписати у такому вигляді: $\mathrm{Cr}_{3} \mathrm{Te}_{4} \rightarrow \mathrm{Cr}^{2+} \mathrm{Cr}_{2}^{3+} \mathrm{Te}_{4}^{2-}$. 3 розрахунку на 1 атом телуру вираз прийме вигляд: $\mathrm{Cr}_{\frac{3}{4}} \mathrm{Te} \rightarrow \mathrm{Cr}_{\frac{1}{4}}^{2+} \mathrm{Cr}_{\frac{2}{4}}^{3+} \mathrm{Te}^{2-}$.

Отже, легуючий кластер запишемо таким чином:

$\mathrm{V}_{\mathrm{Pb}}^{\prime \prime} \mathrm{V}_{\mathrm{Te}}^{\bullet \bullet}+\mathrm{Cr}_{\frac{1}{4}}^{\bullet \bullet} \mathrm{Cr}_{\frac{2}{4}}^{\bullet \bullet \bullet} \mathrm{Te}^{\prime \prime} \rightarrow$
$\left[\mathrm{Cr}_{\frac{2}{4}}^{\bullet} \mathrm{Cr}_{\frac{1}{4}}^{\times} \mathrm{V}_{\frac{1}{4}}^{\prime \prime}\right]_{\mathrm{Pb}} \mathrm{Te}_{\mathrm{Te}}^{\mathrm{x}}$

Суперпозиція (1) 3 легуючим кластером (10) утворить кристалоквазіхімічну формулу твердого розчину n-PbTe- $\mathrm{Cr}_{3} \mathrm{Te}_{4}$ :

$(1-\mathrm{x})\left\{\left[\mathrm{Pb}_{1-\alpha}^{\mathrm{x}}\right]_{\mathrm{Pb}}\left[\mathrm{Te}_{1-\alpha}^{\mathrm{x}}\right]_{\mathrm{Te}}\left(\mathrm{Pb}_{\alpha}^{\bullet}\right)_{\mathrm{i}}+\alpha \mathrm{e}^{\prime}\right\}+$

$\mathrm{x}\left\{\left[\mathrm{Cr}_{\frac{2}{4}}^{\bullet} \mathrm{Cr}_{\frac{1}{4}}^{\times} \mathrm{V}_{\frac{1}{4}}^{\prime /}\right] \mathrm{Te}_{\mathrm{Te}}^{\mathrm{x}}\right\} \rightarrow$

$\left[\mathrm{Pb}_{(1-\alpha)(1-\mathrm{x})}^{\mathrm{x}} \mathrm{Cr}_{\frac{1}{2} \mathrm{x}}^{\bullet} \mathrm{Cr}_{\frac{1}{4} \mathrm{x}}^{\times} \mathrm{V}_{\frac{1}{4} \mathrm{x}}^{\prime \prime}\right]_{\mathrm{Pb}}\left[\mathrm{Te}_{(1-\alpha)(1-\mathrm{x})+\mathrm{x}}^{\mathrm{x}}\right]_{\mathrm{Te}} \times$

$\left(\mathrm{Pb}_{\alpha(1-\mathrm{x})}^{\bullet}\right)_{\mathrm{i}}+\alpha(1-\mathrm{x}) \mathrm{e}^{\prime}$ де $\mathrm{x}$ - мольна частка $\mathrm{Cr}_{3} \mathrm{Te}_{4}$ у твердому розчині.

Аналогічно для твердих розчинів p-PbTe- $\mathrm{Cr}_{3} \mathrm{Te}_{4}$ :

$(1-\mathrm{x})\left\{\left[\mathrm{Pb}_{1-\beta}^{\mathrm{x}} \mathrm{V}_{\beta}^{\prime}\right]_{\mathrm{Pb}} \mathrm{Te}_{\mathrm{Te}}^{\mathrm{x}}+\beta \mathrm{h}^{\bullet}\right\}+$

$\mathrm{x}\left\{\left[\begin{array}{cc}\mathrm{Cr}_{\frac{2}{4}}^{\bullet} & \mathrm{Cr}_{\frac{1}{4}}^{\times} \mathrm{V}_{\frac{1}{4}}^{\prime \prime}\end{array}\right]_{\mathrm{Pb}} \mathrm{Te}_{\mathrm{Te}}^{\mathrm{x}}\right\} \rightarrow$

$\left[\mathrm{Pb}_{(1-\beta)(1-\mathrm{x})}^{\mathrm{x}} \mathrm{Cr}_{\frac{1}{2} \mathrm{x}}^{\bullet} \mathrm{Cr}_{\frac{1}{4} \mathrm{x}}^{\times} \mathrm{V}_{\frac{1}{4} \mathrm{x}}^{\prime \prime} \mathrm{V}_{\beta(1-\mathrm{x})}^{\prime}\right]_{\mathrm{Pb}} \mathrm{Te}_{\mathrm{Te}}^{\mathrm{x}}+$

$\beta(1-\mathrm{x}) \mathrm{h}^{\bullet}$

\section{Концентрації дефектів і носіїв заряду}

Запропоновані механізми легування плюмбум телуриду хромом дають можливість визначити як концентрації окремих точкових дефектів, так i холлівської концентрації носіїв заряду від величини початкового відхилення від стехіометричного складу $\alpha, \beta$ у базовій сполуці PbTe i концентрації легуючої сполуки (х). Так, згідно (5), повне рівняння електронейтральності для $\mathrm{n}-\mathrm{PbTe} \mathrm{Cr}$ можна записати так:

$n=\mathrm{q}_{\mathrm{Cr}_{\mathrm{Pb}}^{\cdot}}\left[\mathrm{Cr}_{\mathrm{Pb}}^{\bullet}\right]+\mathrm{q}_{\mathrm{Pb}_{\mathrm{i}}^{\bullet}}\left[\mathrm{Pb}_{\mathrm{i}}^{\bullet}\right]+\mathrm{q}_{\mathrm{V}_{\mathrm{Te}}} \cdot\left[\mathrm{V}_{\mathrm{Te}}^{\bullet \bullet}\right]$

де $\quad n=\mathrm{A}(\alpha+(3-\alpha-\mathrm{z}) \mathrm{x}), \quad\left[\mathrm{Cr}_{\mathrm{Pb}}^{\bullet}\right]=\mathrm{A}(1-\mathrm{z}) \mathrm{x}$,

$\left[\mathrm{Pb}_{\mathrm{i}}^{\bullet}\right]=\mathrm{A} \alpha(1-\mathrm{x}), \quad\left[\mathrm{V}_{\mathrm{Te}}^{\bullet \bullet}\right]=\mathrm{Ax}, \quad \mathrm{q}_{\mathrm{Cr}_{\mathrm{pb}}^{\bullet}} \approx \mathrm{q}_{\mathrm{Cr}_{\mathrm{i}}^{\bullet}} \approx 1$,

$\mathrm{q}_{\mathrm{V}_{\mathrm{Te}}} \approx 2$, де $\mathrm{A}=\frac{2 Z}{a^{3}}, \mathrm{Z}-$ кількість структурних одиниць в елементарній комірці, $a$ - параметр гратки. Аналогічним чином запишемо для p-PbTe:Cr:

$n+\mathrm{q}_{\mathrm{V}_{\mathrm{Pb}}^{\prime}}\left[\mathrm{V}_{\mathrm{Pb}}^{\prime}\right]=\mathrm{p}+\mathrm{q}_{\mathrm{Cr}_{\mathrm{Pb}}^{\cdot}}\left[\mathrm{Cr}_{\mathrm{Pb}}^{\bullet}\right]+\mathrm{q}_{\mathrm{V}_{\mathrm{Te}}^{. .}}\left[\mathrm{V}_{\mathrm{Te}}^{\bullet \bullet}\right]$

де $n=\mathrm{A}(3-\mathrm{z}) \mathrm{x}, p=\mathrm{A} \beta(1-\mathrm{x}),\left[\mathrm{Cr}_{\mathrm{Pb}}^{\bullet}\right]=\mathrm{A}(1-\mathrm{z}) \mathrm{x}$, $\left[\mathrm{V}_{\mathrm{Pb}}^{\prime}\right]=\mathrm{A} \beta(1-\mathrm{x}), \quad\left[\mathrm{V}_{\mathrm{Te}}^{\bullet \bullet}\right]=\mathrm{Ax}, \quad \mathrm{q}_{\mathrm{Cr}_{\mathrm{Pb}}^{\bullet}} \approx \mathrm{q}_{\mathrm{V}_{\mathrm{Pb}}^{\prime}} \approx 1$, $\mathrm{q}_{\mathrm{V}_{\mathrm{Te}}} \approx 2$.

Для твердих розчинів n-PbTe-CrTe, згідно (8), рівняння повної електронейтральності буде:

$n=\mathrm{q}_{\mathrm{Cr}_{\mathrm{Pb}}^{\cdot}}\left[\mathrm{Cr}_{\mathrm{Pb}}^{\bullet}\right]+\mathrm{q}_{\mathrm{Pb}_{\mathrm{i}}^{\bullet}}\left[\mathrm{Pb}_{\mathrm{i}}^{\bullet}\right]$

де $n=\mathrm{A}(\alpha+(1-\alpha-\mathrm{z}) \mathrm{x}), \quad\left[\mathrm{Cr}_{\mathrm{Pb}}^{\bullet}\right]=\mathrm{A}(1-\mathrm{z}) \mathrm{x}$, $\left[\mathrm{Pb}_{\mathrm{i}}^{\bullet}\right]=\mathrm{A} \alpha(1-\mathrm{x}), \mathrm{q}_{\mathrm{Cr}_{\mathrm{Pb}}^{\bullet}} \approx \mathrm{q}_{\mathrm{Pb}_{\mathrm{i}}^{\bullet}} \approx 1$.

Для $\mathrm{p}-\mathrm{Pb}_{1-\mathrm{x}} \mathrm{Cr}_{\mathrm{x}} \mathrm{Te} \mathrm{відповідно:}$

$n+\mathrm{q}_{\mathrm{V}_{\mathrm{Pb}}^{\prime}}\left[\mathrm{V}_{\mathrm{Pb}}^{\prime}\right]=\mathrm{p}+\mathrm{q}_{\mathrm{Cr}_{\mathrm{Pb}}^{\cdot}}\left[\mathrm{Cr}_{\mathrm{Pb}}^{\bullet}\right]$

де $n=\mathrm{A}(1-\mathrm{z}) \mathrm{x}, p=\mathrm{A} \beta(1-\mathrm{x}),\left[\mathrm{Cr}_{\mathrm{Pb}}^{\bullet}\right]=\mathrm{A}(1-\mathrm{z}) \mathrm{x}$, $\left[\mathrm{V}_{\mathrm{Pb}}^{\prime}\right]=\mathrm{A} \beta(1-\mathrm{x}), \mathrm{q}_{\mathrm{Cr}_{\mathrm{Pb}}^{\cdot}} \approx \mathrm{q}_{\mathrm{V}_{\mathrm{Pb}}^{\prime}} \approx 1$.

Згідно (11), повне рівняння електронейтральності для n-PbTe- $\mathrm{Cr}_{3} \mathrm{Te}_{4}$ можна записати таким чином: 
$n+\mathrm{q}_{\mathrm{V}_{\mathrm{Pb}}^{\prime \prime}}\left[\mathrm{V}_{\mathrm{Pb}}^{\prime \prime}\right]=\mathrm{q}_{\mathrm{Cr}_{\mathrm{Pb}}^{\bullet}}\left[\mathrm{Cr}_{\mathrm{Pb}}^{\bullet}\right]+\mathrm{q}_{\mathrm{Pb}_{\mathrm{i}}} \cdot\left[\mathrm{Pb}_{\mathrm{i}}^{\bullet}\right]$

де $n=\mathrm{A} \alpha(1-\mathrm{x}), \quad\left[\mathrm{V}_{\mathrm{Pb}}^{\prime \prime}\right]=\frac{1}{4} \mathrm{Ax}, \quad\left[\mathrm{Cr}_{\mathrm{Pb}}^{\bullet}\right]=\frac{1}{2} \mathrm{Ax}$,

$\left[\mathrm{Pb}_{\mathrm{i}}^{\bullet}\right]=\mathrm{A} \alpha(1-\mathrm{x}), \mathrm{q}_{\mathrm{Cr}_{\mathrm{Pb}}^{\bullet}} \approx \mathrm{q}_{\mathrm{Pb}_{\mathrm{i}}^{\bullet}} \approx 1, \mathrm{q}_{\mathrm{V}_{\mathrm{Pb}}^{\prime \prime}} \approx 2$.

Аналогічним чином запишемо для p-PbTe- $\mathrm{Cr}_{3} \mathrm{Te}_{4}$ :

$\mathrm{q}_{\mathrm{V}_{\mathrm{Pb}}^{\prime \prime}}\left[\mathrm{V}_{\mathrm{Pb}}^{\prime \prime}\right]+\mathrm{q}_{\mathrm{V}_{\mathrm{Pb}}^{\prime}}\left[\mathrm{V}_{\mathrm{Pb}}^{\prime}\right]=\mathrm{p}+\mathrm{q}_{\mathrm{Cr}_{\mathrm{Pb}}^{\cdot}}\left[\mathrm{Cr}_{\mathrm{Pb}}^{\bullet}\right]$

де $p=\mathrm{A} \beta(1-\mathrm{x}),\left[\mathrm{V}_{\mathrm{Pb}}^{\prime \prime}\right]=\frac{1}{4} \mathrm{Ax},\left[\mathrm{Cr}_{\mathrm{Pb}}^{\bullet}\right]=\frac{1}{2} \mathrm{Ax}$,

$\left[\mathrm{V}_{\mathrm{Pb}}^{\prime}\right]=\mathrm{A} \beta(1-\mathrm{x}), \mathrm{q}_{\mathrm{Cr}_{\mathrm{Pb}}} \approx \mathrm{q}_{\mathrm{V}_{\mathrm{Pb}}^{\prime}} \approx 1, \mathrm{q}_{\mathrm{V}_{\mathrm{Pb}}^{\prime \prime}} \approx 2$.

Результати проведених розрахунків концентрації носіїв та точкових дефектів наведено на Рис. 1-4.

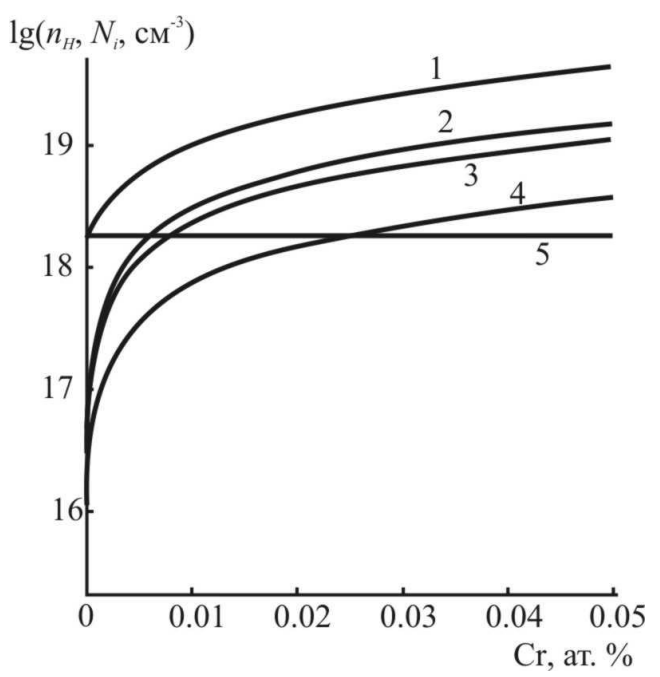

a)

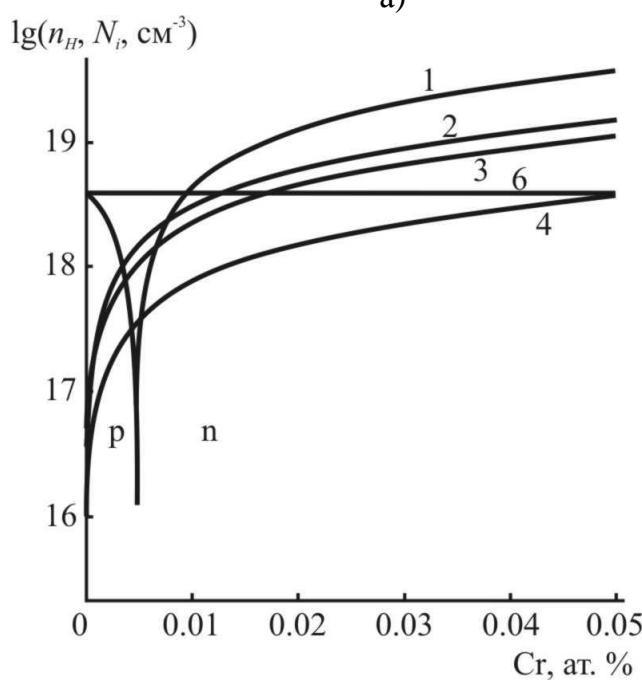

б)

Рис. 1 Залежність холлівської концентрації $\left(1-n_{H}\right)$ та концентрації точкових дефектів $\left(N_{i}\right)$ для кристалів n-PbTe: $\mathrm{Cr}$ (а) $(\alpha=0,006$ ат. \%) i p-PbTe:Cr (б) $(\beta=0,013$ ат. \%) від вмісту домішки $\mathrm{Cr}$ $\left(N_{i}: 2-\left[\mathrm{V}_{\mathrm{Te}}^{2+}\right], 3-\left[\mathrm{Cr}_{\mathrm{Pb}}^{+}\right], 4-\left[\mathrm{Cr}_{\mathrm{Pb}}^{0}\right]\right.$, $\left.5-\left[\mathrm{Pb}_{\mathrm{i}}^{+}\right], 6-\left[\mathrm{V}_{\mathrm{Pb}}^{-}\right]\right)$.

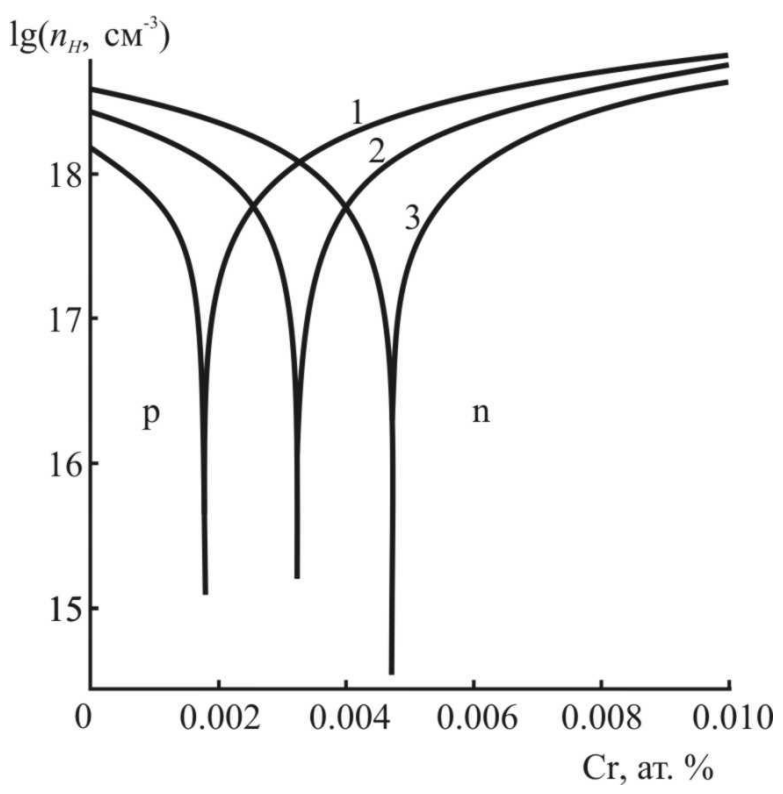

Рис. 2 Розраховані значення холлівської концентрації носіїв заряду $\left(n_{H}\right)$ від вмісту домішки $\mathrm{Cr}$ в кристалах $\mathrm{p}-\mathrm{PbTe}: \mathrm{Cr}$ для різних значень величини початкового відхилення від стехіометрії на боці Те $(\beta$, ат. \%): 0,005(1), 0,009(2), 0,013(3).

\section{Результати та їх обговорення}

На основі проведених розрахунків встановлено, що для p-PbTe:Cr при малих кількостях домішки відбувається зменшення концентрації основних носіїв, конверсія типу провідності та зростання концентрації електронів при подальшому зростанні вмісту Cr (Рис. 1(б) - крива 1), що явно ілюструє донорну дію хрому. При чому крива p-n-переходу змішується в бік більших концентрацій домішки при збільшенні величини початкового відхилення від стехіометрії ( $\beta$ ) (Рис. 2). Зауважимо, що таке зміщення в даному випадку $є$ несуттєвим. Для n-PbTe:Cr концентрація основних носіїв струму різко зростає зі збільшенням вмісту $\mathrm{Cr}$ (Рис. 1(а) крива 1). Особливості, що спостерігаємо у зміні концентрації носіїв заряду, пов'язані 3 характерними співвідношеннями між окремими точковими дефектами. Так, для $\mathrm{PbTe}: \mathrm{Cr}$ домінуючими дефектами $є$ іонізовані атоми хрому на місці плюмбуму $\mathrm{Cr}_{\mathrm{Pb}}^{+}$(Рис. 1 - крива 3) та вакансії телуру $\mathrm{V}_{\mathrm{Te}}^{2+}$ (Рис. 1 - крива 2), концентрація яких різко зростає 3 ростом концентрації Cr. Крім того, якщо для n-PbTe:Cr домінує міжвузловий плюмбум $\mathrm{Pb}_{\mathrm{i}}^{+}$(Рис. 1(a) крива 5), то для p-PbTe:Cr - вакансії в катіонній підгратці $\mathrm{V}_{\mathrm{Pb}}^{-}$(Рис. 1(б) - крива 6), концентрація яких змінюється незначно від вмісту домішки. 


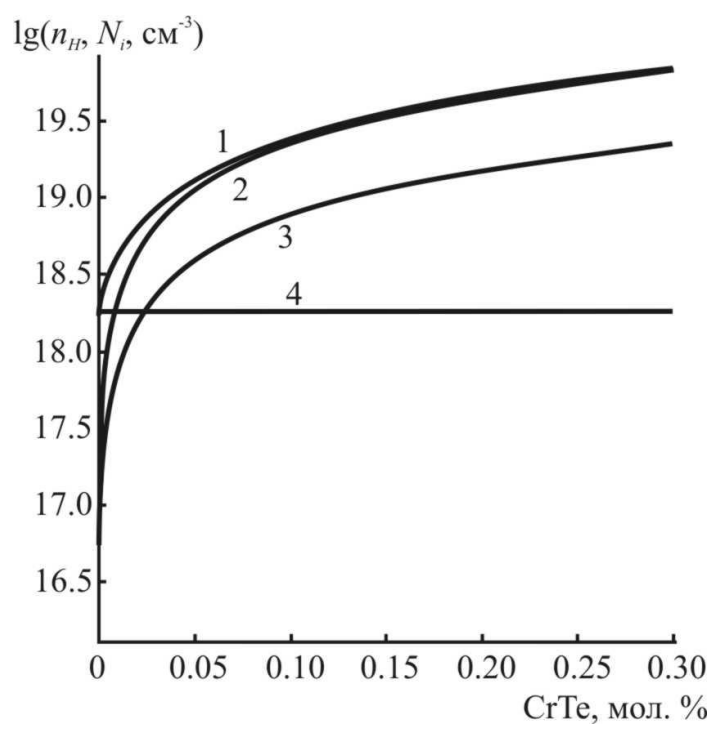

a)

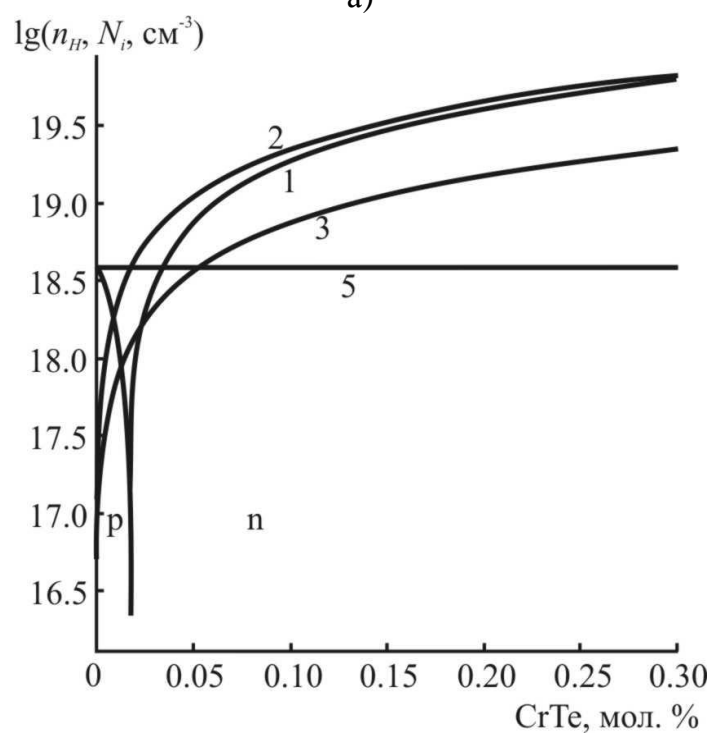

б)

Рис. 3 Залежність холлівської концентрації $\left(1-n_{H}\right)$ та концентрації точкових дефектів $\left(N_{i}\right)$ твердих розчинів n-PbTe-CrTe (a) $(\alpha=0,006$ ат. $\%) \quad$ i $\quad$ p-PbTe-CrTe

$\left(\beta=0,013\right.$ ат. \%) від складу $\left(N_{i}: 2-\left[\mathrm{Cr}_{\mathrm{Pb}}^{+}\right]\right.$,

$\left.3-\left[\mathrm{Cr}_{\mathrm{Pb}}^{0}\right], 4-\left[\mathrm{Pb}_{\mathrm{i}}^{+}\right], 5-\left[\mathrm{V}_{\mathrm{Pb}}^{-}\right]\right)$.

Для $\mathrm{p}-\mathrm{Pb}_{1-\mathrm{x}} \mathrm{Cr}_{\mathrm{x}} \mathrm{Te}$ спочатку відбувається зменшення концентрації основних носіїв, конверсія типу провідності та подальше зростання концентрації електронів (Рис. 3(б) - крива 1), що підтверджує донорну дію $\mathrm{CrTe}$. Для $\mathrm{n}-\mathrm{Pb}_{1-\mathrm{x}} \mathrm{Cr}_{\mathrm{x}} \mathrm{Te}$ концентрація основних носіїв струму зі збільшенням вмісту CrTe зростає (Рис. 3(а) - крива 1). Особливості, що можна спостерігати у зміні холлівської концентрації носіїв (Рис. 3), зумовлені характерними співвідношеннями між окремими точковими дефектами. Так, для твердих розчинів $\mathrm{Pb}_{1-\mathrm{x}} \mathrm{Cr}_{\mathrm{x}}$ Те суттєвий вплив на концентрацію носіїв мають йонізовані атоми хрому на місці плюмбуму (Рис. 3 - крива 2), концентрація яких різко зростає 3 ростом концентрації CrTe. Крім того, якщо для

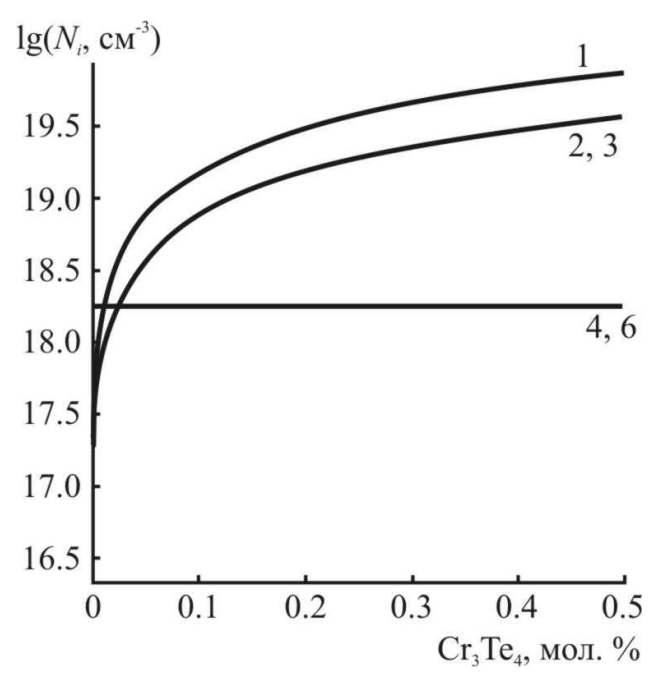

a)

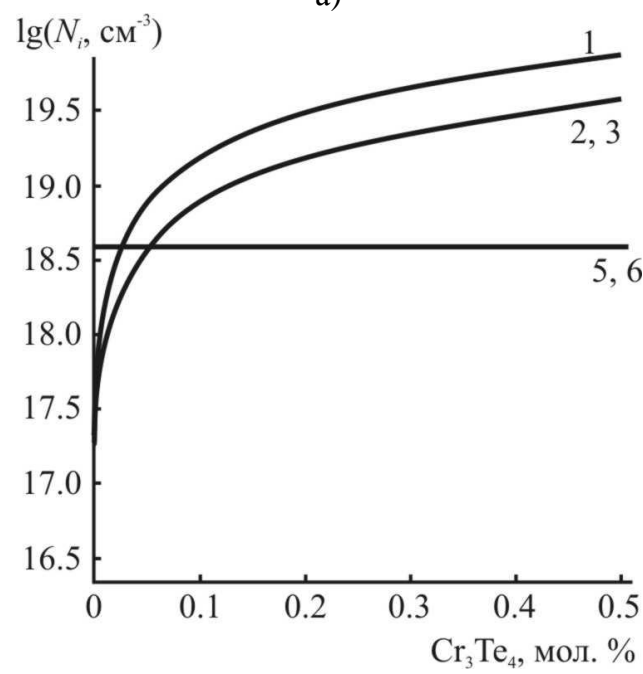

б)

Рис. 4 Залежність холлівської концентрації $\left(6-n_{H}\right)$ та концентрації точкових дефектів $\left(N_{i}\right)$ для кристалів n-PbTe- $\mathrm{Cr}_{3} \mathrm{Te}_{4} \quad$ (a) $\left(\alpha=0,006\right.$ ат. \%) та $\quad$ p-PbTe- $\mathrm{Cr}_{3} \mathrm{Te}_{4} \quad$ (б) $\left(\beta=0,013\right.$ ат. \%) від складу (х) $\left(N_{i}: 1\right.$ $\left[\mathrm{Cr}_{\mathrm{Pb}}^{+}\right], 2-\left[\mathrm{V}_{\mathrm{Pb}}^{2-}\right], 3-\left[\mathrm{Cr}_{\mathrm{Pb}}^{0}\right], 4-\left[\mathrm{Pb}_{\mathrm{i}}^{+}\right], 5-$ $\left.\left[\mathrm{V}_{\mathrm{Pb}}^{-}\right]\right)$.

$\mathrm{n}-\mathrm{Pb}_{1-\mathrm{x}} \mathrm{Cr}_{\mathrm{x}} \mathrm{Te}$ домінує міжвузловий плюмбум $\mathrm{Pb}_{\mathrm{i}}^{+}$ (Рис. 3(a) - крива 4), то для $\mathrm{p}-\mathrm{Pb}_{1-\mathrm{x}} \mathrm{Cr}_{\mathrm{x}} \mathrm{Te}-$ катіонні вакансії $\mathrm{V}_{\mathrm{Pb}}^{-}$(Рис. 3(б) - крива 5), концентрація яких зі збільшенням вмісту домішки змінюється незначно.

Для $\mathrm{n}-\mathrm{PbTe}-\mathrm{Cr}_{3} \mathrm{Te}_{4}$ концентрація носіїв струму iз збільшенням вмісту $\mathrm{Cr}_{3} \mathrm{Te}_{4}$ слабо зменшується (Рис. 4 - крива 6). Зниження концентрації носіїв підтверджується експериментальними даними. Аналогічна картина - незначне зменшення концентрації основних носіїв - характерна для $\mathrm{p}-\mathrm{PbTe}-\mathrm{Cr}_{3} \mathrm{Te}_{4}$. Для випадку $\mathrm{n}-\mathrm{PbTe}-\mathrm{Cr}_{3} \mathrm{Te}_{4}$ суттєвий вплив на концентрацію носіїв мають двозарядні вакансії плюмбуму $\mathrm{V}_{\mathrm{Pb}}^{2-}$ та іонізовані атоми хрому на місці плюмбуму $\mathrm{Cr}_{\mathrm{Pb}}^{+}$, 
концентрація яких різко зростає 3 ростом вмісту $\mathrm{Cr}_{3} \mathrm{Te}_{4}$ (Рис. 4(а) - криві 2 та 1 відповідно). Концентрація міжвузлового плюмбуму $\mathrm{Pb}_{\mathrm{i}}^{+}$ змінюється незначно (Рис. 4(а) - крива 4). Значне зростання концентрації домішкових дефектів спостерігаємо також для p-PbTe-Cr $\mathrm{Te}_{4}$ (Рис. 4(б) криві 1,3). Відмітимо, що в цьому випадку концентрація однозарядних катіонних вакансій слабо зменшується з ростом мольної частки $\mathrm{Cr}_{3} \mathrm{Te}_{4}$ (Рис. 4(б) - крива 5).

Оскільки іонний радіус хрому значно менший, ніж іонний радіус плюмбуму: $r_{\mathrm{Cr}^{3+}}<r_{\mathrm{Cr}^{2+}}<r_{\mathrm{Pb}^{2+}}$ $\left(r_{\mathrm{Cr}^{2+}}=0,83 \AA\right.$, $r_{\mathrm{Pb}^{2+}}=1,26 \AA$ ), то заміщення хромом катіонних вакансій зумовлюватиме спостережуване зменшення параметра гратки.

\section{Висновки}

1. Запропоновано моделі точкових дефектів i кристалоквазіхімічні формули як легованих хромом кристалів РbTe, так і твердих розчинів $\mathrm{PbTe}-\mathrm{CrTe}$ та $\mathrm{PbTe}-\mathrm{Cr}_{3} \mathrm{Te}_{4}$.

2. Розраховано залежності концентрації точкових дефектів, вільних носіїв та холлівської концентрації носіїв струму від вмісту легуючих сполук $\left(\mathrm{Cr}, \mathrm{CrTe}, \mathrm{Cr}_{3} \mathrm{Te}_{4}\right)$ для кристалів n- i p-PbTe. 3. Визначено умови формування термодинамічних p-n-переходів для кристалів p-PbTe:Cr та p-PbTeCrTe.

4. Встановлено, що у твердих розчинах домінуючими $\epsilon$ як власні точкові дефекти $\left(\mathrm{V}_{\mathrm{Te}}^{2+}, \mathrm{V}_{\mathrm{Pb}}^{2-}, \mathrm{Pb}_{\mathrm{i}}^{+}\right)$, так $\mathrm{i}$ домішкові $\left(\mathrm{Cr}_{\mathrm{Pb}}^{+}, \mathrm{Cr}_{\mathrm{Pb}}^{0}\right)$, перерозподіл яких визначає фізико-хімічні властивості.

\section{Подяка}

Робота виконується в рамках наукових проектів МОН України (державні реєстраційні номери: 0107U006768 та 0109U007537).

\section{Літературні посилання}

[1] Н.Х. Абрикосов, Л.М. Шалимова, Полупроводниковые материаль на основе соединений $A^{I V} B^{V I}$, Наука, Москва, 1975, 196 с.

[2] В.М. Шперун, Д.М. Фреїк, Р.I. Запухляк, Термоелектрика телуриду свиниюю та його аналогів, Плай, Івано-Франківськ, 2000, 250 с.

[3] Д.М. Фреїк, В.В. Прокопів, М.О. Галущак, М.В. Пиц, Г.Д. Матеїк, Кристалохімія $i$ термодинаміка дефектів у сполуках $A^{I V} B^{V I}$, Плай, Івано-Франківськ, 1999, 164 с.

[4] T. Leo Ngai, D. Marshall, R.C. Sharma, Y.A. Chang, Monatsh. Chem. 118(3) (1987) 277-300.

[5] Т.И. Конешова, К.В. Еремин, В.М. Новоторцев, Неорг. Матер. 31(12) (1995) 1525-1529.

[6] П.В. Вертелецкий, В.П. Зломанов, О.И. Тананаева, Неорг. Матер. 34(4) (1998) 400405.

[7] H. Ipser, K.L. Komarek, J. Less-Common Met. 92(2) (1983) 265.

[8] С.А. Ставрианидис, А.А. Куция, Р.А. Маркелия, Г.А. Кейян, Ю.А. Мальцев, Неорг. Maтер. 22(5) (1986) 744-746.

[9] Г.А. Кейян, А.А. Куция, Р.А. Маркелия, С.А. Ставрианидис, Неорг. Матер. 24(4) (1988) 588-590.

[10] А.Н. Васильев, Ю.П. Гайдуков, В.П. Зломанов, В.Н. Никифоров, О.Н. Тананаева, Hеорг. Mатер. 24(2) (1988) 227-229.

[11] Z. Golaski, K. Godwod, J. Majewski, G. Jasiotek, J. Cryst. Growth. 84(3) (1987) 455-459.

[12] П.В. Вертелецкий, Т.А. Кузнецова, В.П. Зломанов, О.И. Тананаева, Электрон. Техн., Сер. Материаль 4 (241) (1989) 66-70.

[13] С.С. Лисняк, Неорг. Матер. 32(2) (1992) 1913-1917. 